\title{
Les «boîtes à bébé», éthiquement acceptables?
}

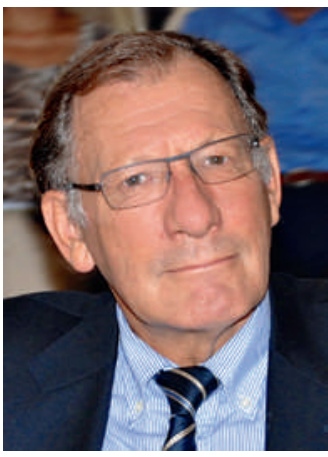

Jean Martin
Le sujet de l'abandon d'enfant dans des boîtes à bébé (Babyfenster) a repris de l'actualité récemment. Une telle «fenêtre» a été ouverte à Davos en juin 2012 et d'autres endroits s'y intéressent. Surtout, à la fin de 2012, les Grands Conseils valaisan et bernois ont proposé à leurs gouvernements de suivre ce mouvement. Rappelons aussi un article récent à ce sujet du pédiatre Stephan Rupp [1].

La grande presse [2] note que, selon une enquête Isopublic, une large majorité de nos concitoyens y seraient favorables. Délicat dans ces conditions de dire son avis que ces «boîtes» sont une fausse bonne idée... Dans son intéressant texte traitant des sept enfants (en neuf ans) déposés à Einsiedeln, notre confrère Rupp toutefois se concentre sur les aspects objectifs et médicaux/physiques. Sans qu'il s'agisse ici de mettre en cause la bonté des intentions des promoteurs (bonté qui pourrait être un motif explicatif d'une opinion populaire positive), il ne paraît simplement pas possible de se passer d'une évaluation en termes éthiques et juridiques. Cette évaluation m'amène à formuler d'importantes réserves.

Le droit de l'enfant à connaître ses origines - Pourquoi ne pas donner l'enfant pour adoption? Même si chacun comprend qu'une femme qui vient d'accoucher peut dans certains cas tenir à l'anonymat (mais est-ce toujours sa volonté à elle? cf. ci-dessous), on doit absolument considérer l'intérêt de l'enfant, qui peut prévaloir sur celui de la mère. En effet, le droit de l'enfant de connaître ses origines (dans toute la mesure du faisable) est maintenant largement reconnu, y compris dans des lois. L'alternative à l'abandon anonyme du nouveau-né est évidente, c'est l'adoption telle qu'elle est habituellement pratiquée. En fait, le seul risque que la mère évite par l'intermédiaire de la «Babyfenster», c'est que l'enfant veuille et puisse faire sa connaissance lorsqu'il aura 16 ou 18 ans. Or, aujourd'hui, la pesée des intérêts en cause est clairement en faveur de ce droit pour l'enfant.

Une pratique anachronique. Rappelons que l'institution française de l'accouchement sous X, comparable aux plans éthique et pratique à la «boîte à bébé», introduite vers 1600 (avec des «tours» dans des hospices ou églises) et formalisée à la Révolution, a été sérieusement contestée dans le passé récent. La Cour européenne des droits de l'homme cependant a renoncé en 2003 à l'invalider, estimant que «la France n'excède pas la marge d'appréciation qui doit lui être reconnue», mais des observateurs jugent qu'il y avait là, au moins en partie, un égard diplomatique. Aujourd'hui, j'en suis convaincu, un Etat de droit ne peut/doit pas collaborer, vis-à-vis d'un enfant, à l'ac-$$
\text { peut/doit pas collaborer, vis-à-vis d'un enfant, à l'ac- }
$$

tion de cacher/camoufler ses origines. Et encore moins encourager une telle démarche!

Risque de pressions sur la jeune mère. Un des soucis à garder à l'esprit, dont la concrétisation éventuelle est clairement choquante, est que l'abandon du bébé soit le résultat de pressions de l'entourage de la femme - et donc contre l'avis de cette dernière. Ceci sans qu'aucune discussion ou médiation, qui pourrait avoir un effet préventif, soit possible puisque tout se fait dans un complet anonymat. Bien qu'il soit difficile d'obtenir des données à ce sujet, ce risque n'est, de l'avis de professionnelles, pas négligeable [3].

Ne pas noircir le tableau. Certains laissent entendre (ce n'est toutefois pas le cas du Dr Rupp) que les nouveau-nés recueillis seraient décédés si une «boîte» n'avait pas été disponible. En réalité, c'est très invraisemblable. Tout de même, nous sommes en Suisse et le réseau d'aide médico-sociale est dense et diversifié.

Réfléchir au-delà du «bon cour». A mon sens, l'évolution inadéquate qu'il faut éviter est que, de manière simpliste, dans la population voire chez les autorités, la «boîte à bébé» vienne à être considérée comme un progrès. Un signe d'une société libérale et solidaire?! Etant entendu que ceux qui ont mis en place de tels dispositifs ont droit au respect, on peut penser au contraire qu'il s'agit d'une sorte de retour en arrière, à une époque où l'abandon d'enfants et même l'infanticide étaient courants et où on était beaucoup moins sensible aux droits fondamentaux des personnes, y compris ceux des enfants.

Ainsi, je ne peux certainement pas me rallier à la conclusion de notre confrère Rupp (ma traduction de l'allemand) qu'«il serait souhaitable d'ouvrir un plus grand nombre de «Babyfenster〉 dans les hôpitaux, si possible disséminés dans le pays». Il semble admis, je le réalise, que des personnes ou groupes qui croient en leur pertinence ont le droit d'en créer. Il reste que les problématiques d'éthique médicale et sociétale qui y sont liées sont sérieuses et ne peuvent pas être ignorées.

Jean Martin, membre de la rédaction

1 Rupp S. 10 Jahre Einsiedler Babyfenster - ein Rückblick. Bull Méd Suisses. 2012;93(24):918-20.

2 Parmi d'autres: Zuercher C. Les boîtes à bébé sont-elles une solution? Le doute s'installe. 24 Heures (Lau-

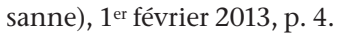

3 Curtet N., Denisart D. Femmes abandonnées, bébés lésés. 24 Heures (Lausanne), $1^{\text {er }}$ février 2013, p. 2. 